\title{
ALTERAÇÕES DIMENSIONAIS EM PEÇAS USINADAS POR ELETROEROSÃO DEVIDO AO SEU BENEFICIAMENTO PRÉVIO*
}

\author{
Daniel Alves Sodré ${ }^{1}$ \\ Eduardo Tramontina ${ }^{2}$ \\ Marcos Tadeu Tavares Pacheco ${ }^{3}$ \\ Willy Ank de Morais ${ }^{4}$
}

\begin{abstract}
Resumo
O processo de eletroerosão por penetração permite a usinagem de componentes metálicos nas mais variadas condições de tratamento termo mecânico e durezas. Entretanto, as tabelas e fórmulas tecnológicas empregadas para a execução deste processo não consideram essas distintas condições do material com uma variável. Desta forma, este trabalho objetivou avaliar a influência dimensional na usinagem via eletroerosão por penetração em componentes com e sem tratamento térmico de têmpera seguida de revenimento. Foram avaliadas amostras de aços ferramenta largamente empregados na indústria, representados pelas normas ABNT NM 122 Graus D6 e 01 e ABNT NM 87 Grau 1045. Observou-se uma variação dimensional de $20 \mu \mathrm{m}$ entre as amostras.

Palavras-chave: Eletroerosão por penetração; Usinagem; Tratamento térmico.

\section{DIMENSIONAL CHANGES IN PARTS MACHINED BY PENETRATION ELECTRICAL DISCHARGE DUE TO THERMAL TREATMENT}

\section{Abstract}

The process of electrical discharge machining (EDM) by penetration, allows manufacture metallic parts in various conditions of thermal treatment and hardness. However, the tables and technological formulas used to implement this process do not consider these different conditions of the material as a variable. Thus, this study aimed to evaluate the dimensional influence on machining components by EDM with and without heat treatment by quenching followed by tempering. It were evaluated industrial largely used tools steels, represent by the standards ABNT NM 122 Grades D6 and O1, and ABNT NM 87 Grade 1045. It was observed a dimensional variation between samples of $20 \mu \mathrm{m}$.

Keywords: EDM by penetration; Machining; Heat treatment.

1 Tecnólogo Mecânico, mestrando em Engenharia Mecânica da UNISANTA e Professor/Instrutor no SENAI Manuel Garcia Filho, Diadema, Brasil (danielsodre@uol.com.br).

2 Tecnólogo Mecânico, mestrando em Engenharia Mecânica, Universidade Santa Cecília, Santos, Brasil (etramontina@hotmail.com).

3 Doutor e Mestre em Física, Engenheiro Elétrico, Professor e Coordenador dos cursos de PósGraduação Stricto Sensu da UNISANTA, Santos, Brasil (marcttadeu@unisanta.br).

4 Doutorando, Mestre, Engenheiro e Técnico em Metalurgia e Materiais, Consultor Técnico na INSPEBRAS, Sócio-Diretor da Willy Ank Soluções Metal-Mecânicas e Professor Adjunto, UNISANTA, Santos, SP, Brasil (willyank@unisanta.br). 


\section{INTRODUÇÃO}

O processo de usinagem por descargas elétricas (Electrical Discharge Machining $E D M)$ ou eletroerosão por penetração é muito utilizado na indústria de ferramentaria para construção de moldes e matrizes de geometrias complexas, cuja usinagem pelos processos convencionais não seria viável [1 e 2]. Esses componentes, fabricados pelo processo de EDM, são empregados como ferramentas e entram na confecção dos mais variados tipos de produtos, desde peças plásticos que compõem um telefone celular até as complexas partes estampadas em aço dos veículos [4].

As tolerâncias dimensionais necessárias para este tipo de usinagem podem chegar à casa dos milésimos de milímetros $(1 \mu \mathrm{m})$, em função da precisão imposta pelo projeto [3]. Esta precisão, obtida durante a usinagem dos moldes, garantirá o perfeito funcionamento de matrizes de conformação (estampos) e a obtenção da qualidade final necessária ao produto.

Durante a usinagem, a garantia de parâmetros como precisão dimensional, rugosidade superficial, tolerância de forma e posição depende, entre outras coisas, do regime de trabalho adotado pelo operador do equipamento de usinagem, que no caso do EDM é função do tipo de material da peça e do eletrodo ferramenta. De acordo com a teoria de eletroerosão, as propriedades mecânicas da peça e do eletrodo ferramenta, têm pouca influência no desempenho do processo, ao contrário do que ocorre com as propriedades físicas, tais como, condutividade térmica e elétrica, a expansão térmica, a temperatura de fusão e de ebulição, que influenciam profundamente no processo [5].

Para definir os parâmetros de usinagem, ou seja, tempo de impulso, tempo de pausa e a amperagem adequados em uma máquina EDM são utilizadas tabelas tecnológicas fornecidas pelos fabricantes das máquinas [6]. Entretanto tais tabelas definem apenas os materiais usados como eletrodo ferramenta, cobre ou grafite, e da peça e ser erodida, por exemplo, um dos diversos tipos de aços. Quanto ao material a ser erodido, as tabelas não especificam a condição de tratamento térmico ao qual a peça foi submetida, tampouco a dureza das mesmas. Assim sendo, o foco deste trabalho foi identificar a influência do beneficiamento, nome tradicionalmente atribuído às operações de tratamento térmico por têmpera e revenimento para ajuste das propriedades mecânicas finais de aços, nas dimensões finais de peças usinadas pelo processo de eletroerosão.

\section{MATERIAIS E MÉTODOS}

Os experimentos foram efetuados em uma máquina de eletroerosão por penetração (EDM) marca Eletro modelo ZNC 400, ilustrada na Figura 1. Como fluído dielétrico foi empregado um óleo composto a base de hidrocarbonetos recomendado pelo fabricante da máquina.

Foram usinados 6 eletrodos ferramentas cilíndricos em cobre eletrolítico com diâmetros de $12,7 \mathrm{~mm}$ ( $1 / 2$ polegada), conforme ilustrados na Figura 2.0 acompanhamento do desgaste foi efetuado pela variação mássica dos eletrodos, averiguada através de uma balança com precisão de 0,01 grama. As dimensões das amostras, após a usinagem, foram aferidas por uma máquina Tridimensional marca Tesa Micro-Hite [8], também ilustrada na Figura 1. 


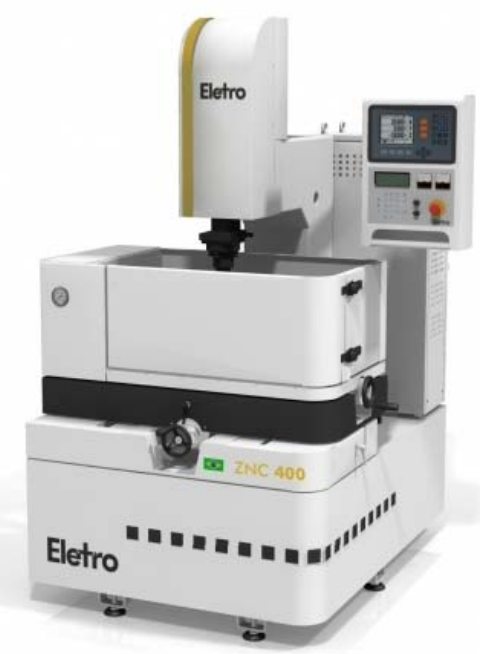

(a)

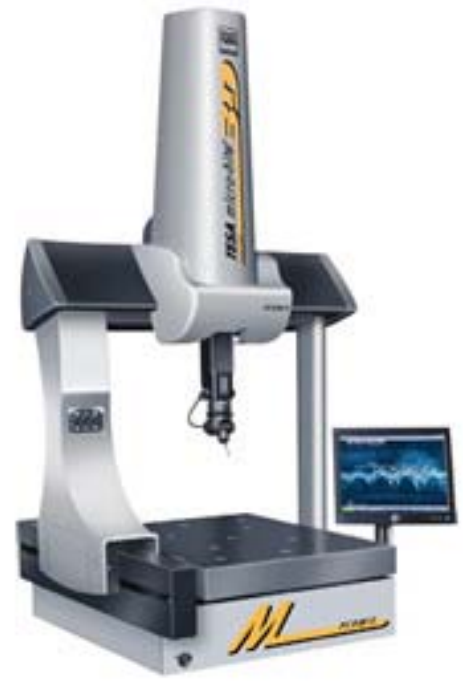

(b)

Figura 1. Equipamentos empregados neste trabalho: (a) máquina de eletroerosão Eletro Modelo ZNC 400 empregada neste trabalho [7] e (b) Máquina de medições tridimensionais Tesa Micro-Hite [8].

Empregaram-se 3 duplas de corpos de prova (CPs) cilíndricos, obtidos de três aço para ferramentas: ABNT NM 122 grau D6 [9], ABNT NM 122 grau O1 [9] e ABNT NM 87 grau 1045 [10]. Um CP de cada aço foi temperado e revenido e o outro foi empregado na condição do material como fornecido, conforme ilustrado na Figura 2.

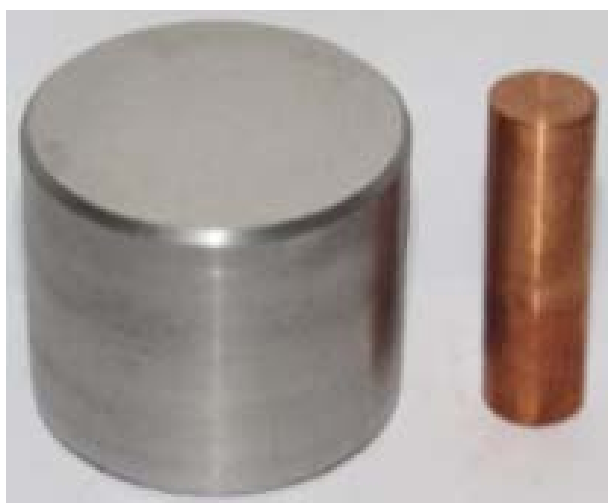

(a)

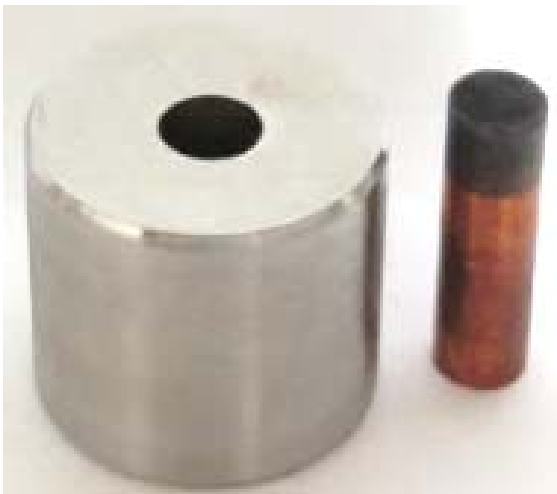

(c)

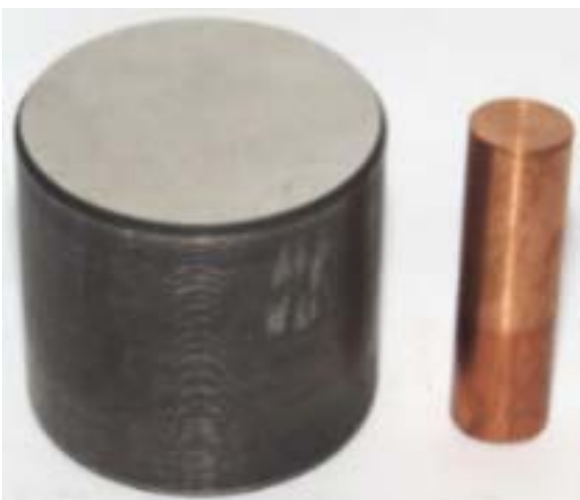

(b)

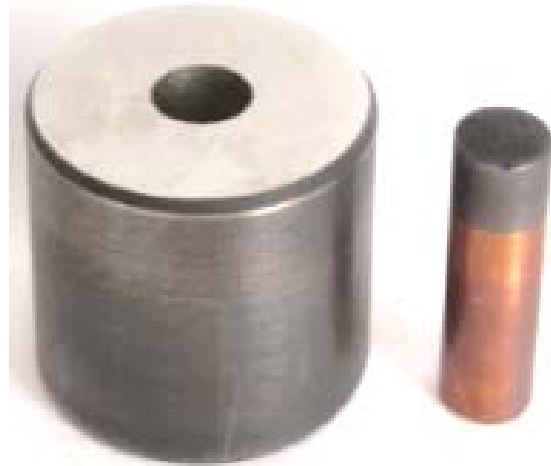

(d)

Figura 2. Amostras de aço ABNT NM 122 Grau D6 [8] e do e eletrodo ferramenta antes e depois da usinagem: (a) condição não tratado e não usinado; (b) condição tratado e não usinado; (c) condição não trado e usinado e (d) condição tratado e usinado. 
As peças foram usinadas aos pares, simultaneamente, sempre do mesmo aço nas duas condições de tratamento, a até uma profundidade de $10 \mathrm{~mm}$. Tanto os eletrodos quanto as amostras formam previamente identificados e levados para a eletroerosão por penetração. Foram empregados os mesmos parâmetros de usinagem tanto para a peça tratada termicamente como para a peça sem tratamento térmico, alguns dos quais estão destacados na Figura 3, foram:

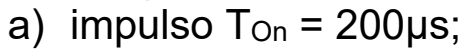

b) quantidade de corrente $l_{e}=3 A$;

c) pausa Toff $=92 \%$;

d) Tempo de erosão = 3s;

e) distância de afastamento $=2 \mathrm{~mm}$.

Esses parâmetros são recomendados pelo manual de operações do equipamento [7] para obter o menor desgaste possível do eletrodo e uma rugosidade de acabamento $R_{\text {máx }}=34 \mu \mathrm{m}$. Após os testes, tanto o desgaste final do eletrodo quanto as dimensões finais dos CPs foram avaliados.

\begin{tabular}{|c|c|c|c|c|c|c|c|c|c|c|c|c|}
\hline \multicolumn{5}{|c|}{ Eletro } & \multicolumn{8}{|c|}{ Manual de Instalação, Operação e Manutenção } \\
\hline \multicolumn{5}{|c|}{$\begin{array}{l}\text { ELETRODO: Cobre ( Eletrolítico ) } \\
\text { PEÇA: Aço }\end{array}$} & \multicolumn{7}{|c|}{$\begin{array}{l}\text { POLARIDADE DO ELETRODO: }(+) \\
\text { TENSÃO: 100V }\end{array}$} & \\
\hline \multicolumn{3}{|c|}{$\begin{array}{l}\text { VALORES } \\
\text { ESTABELECIDOS }\end{array}$} & \multirow{3}{*}{$\begin{array}{l}\text { RUGOSIDADE } \\
\text { Rmax } \\
\mu \mathrm{m}\end{array}$} & \multirow{3}{*}{\begin{tabular}{|l|} 
REMOÇÃO \\
MATERIAL \\
$\mathrm{VW}$ \\
$\mathrm{mm}^{3 / \mathrm{min}}$ \\
\end{tabular}} & \multirow{3}{*}{$\begin{array}{l}\text { DESGASTE } \\
\text { Vv } \\
\%\end{array}$} & \multicolumn{4}{|c|}{$\begin{array}{l}\text { SUBDIMENSIONAMENTO } \\
2 \mathrm{gs}=\mathrm{B}-\mathrm{A}\end{array}$} & \multicolumn{3}{|c|}{$\begin{array}{l}\text { CONDIÇÃO } \\
\text { TESTE }\end{array}$} \\
\hline On & Ie & Dc & & & & PRESSÃ & $(\mathrm{mm})$ & SUCÇÃO & $(\mathrm{mm})$ & Vmin & Iav & Área \\
\hline$\mu \mathrm{s}$ & QT & $\%$ & & & & 2gs max & 2gs & $2 \mathrm{gs} \max$ & $2 \mathrm{gs}$ & Volt & A & $\mathrm{Cm}^{2}$ \\
\hline 200 & 2 & 90 & 30 & 14 & 0.0 & 0.250 & 0.130 & 0.238 & 0.118 & 38 & 7 & 5 \\
\hline 200 & 3 & 92 & 34 & 32 & 0.0 & 0.284 & 0.144 & 0.272 & 0.132 & 35 & 10 & 5 \\
\hline 200 & 4 & 92 & 38 & 60 & 0.3 & 0.314 & 0.156 & 0.303 & 0.145 & 33 & 14 & 10 \\
\hline 200 & 5 & 92 & 43 & 86 & 0.9 & 0.347 & 0.166 & 0.340 & 0.159 & 32 & 17 & 10 \\
\hline
\end{tabular}

Figura 3. Dados tecnológicos fornecida pelo fabricante da máquina [7].

\section{RESULTADOS E DISCUSSÃO}

As características inicias dos corpos de prova e dos eletrodos ferramenta empregados estão mostrados na Tabela 1. Após a usinagem, não formam encontrados diferenças significativas de massa nos eletrodos em até $0,01 \mathrm{~g}$. Essa condição, que levou a um desgaste de $0,0 \%$, coincide com as indicações do fabricante [7].

Tabela 1. Dados coletados das amostras de aço e dos eletrodos ferramenta antes da usinagem.

\begin{tabular}{|c|c|c|c|c|c|c|c|}
\hline \multicolumn{5}{|c|}{ Dados das amostras de aço ABNT NM 122[9] e NM 87[11] } & \multicolumn{3}{|c|}{ Dados dos eletrodos ferramenta } \\
\hline $\mathrm{N}^{\circ}$ & $\begin{array}{c}\text { Dimensões } \\
(\mathrm{mm})\end{array}$ & Aço & Condição & $\begin{array}{c}\text { Dureza } \\
\text { (HRc) }\end{array}$ & $\mathrm{N}^{\circ}$ & $\begin{array}{c}\text { Diâmetro } \\
\text { (mm) }\end{array}$ & $\begin{array}{c}\text { Peso } \\
\text { médio }(\mathrm{g})\end{array}$ \\
\hline 1 & \multirow{6}{*}{$\begin{array}{c}\text { Diâmetro: } 45 \\
\text { Altura: } 32\end{array}$} & \multirow{2}{*}{ D6 [9] } & Tratado & 59 a 61 & 1 & 12,704 & 39,66 \\
\hline 2 & & & Como Receb. & $<20$ & 2 & 12,703 & 40,33 \\
\hline 3 & & \multirow{2}{*}{$1045[11]$} & Tratado & 55 a 58 & 3 & 12,704 & 39,33 \\
\hline 4 & & & Como Receb. & $<20$ & 4 & 12,703 & 39,00 \\
\hline 5 & & \multirow{2}{*}{ O1 [9] } & Tratado & 56 a 58 & 5 & 12,703 & 40,66 \\
\hline 6 & & & Como Receb. & $<20$ & 6 & 12,702 & 39,33 \\
\hline
\end{tabular}


A Tabela 2 apresenta os dados obtidos após a usinagem das amostras. As dimensões finais usinadas nas amostras de aço na condição sem tratamento térmico (como recebidas) apresentaram-se $18 \mu \mathrm{m}$ maiores do que nas amostras de aço que haviam sido tratadas termicamente. Mesmo uma variação dimensional de $18 \mu \mathrm{m}$ pode afetar a qualidade dos ajustes de moldes e matrizes usinados e consequentemente a qualidade final do produto.

Tabela 2. Dados coletados das amostras de aço ABNT NM 122 [9] e NM 87 [11] após a usinagem.

\begin{tabular}{|c|c|c|c|c|c|c|}
\hline $\mathbf{N}^{\circ}$ & Aço & Condição & $\begin{array}{l}\text { Diâmetro do furo } \\
\text { usinado (mm) }\end{array}$ & $\begin{array}{l}\text { Diferença } \\
\qquad(\mu \mathrm{m})\end{array}$ & $\begin{array}{l}\text { Rugosidade } \\
\qquad(\mu \mathrm{m})\end{array}$ & $\begin{array}{l}\text { Tempo de } \\
\text { usinagem } \\
\text { (min.) }\end{array}$ \\
\hline 1 & \multirow{2}{*}{ D6 [9] } & Tratado & 12,941 & \multirow{2}{*}{18} & 33 & 41 \\
\hline 2 & & Como Receb. & 12,959 & & 34 & 43 \\
\hline 3 & \multirow{2}{*}{$\begin{array}{l}1045 \\
{[11]}\end{array}$} & Tratado & 12,924 & \multirow{2}{*}{18} & 32 & 40 \\
\hline 4 & & Como Receb. & 12,942 & & 34 & 42 \\
\hline 5 & \multirow{2}{*}{ O1 [9] } & Tratado & 12,923 & \multirow{2}{*}{19} & 32 & 40 \\
\hline 6 & & Como Receb. & 12,942 & & 33 & 43 \\
\hline
\end{tabular}

Também foi possível observar, pelos dados obtidos e apresentados na Tabela 2, uma coerência dos valores de rugosidade e da taxa de remoção de material com os dados previstos pelo fabricante do equipamento (Figura 3).

Para garantir a precisão dimensional da peça, preparadores de uma máquina e eletroeorosão têm como recurso a utilização de tabelas tecnológicas fornecidas pelos fabricantes e cálculos de medida final do eletrodo em função do GAP $(G)$ da máquina. O GAP é a distância entre o eletrodo e a peça que, para o caso estudado e conforme a Figura 3, variou entre 67 a $124 \mu \mathrm{m}(2 \mathrm{GAP}=132$ a $284 \mu \mathrm{m})$.

Com relação as tabelas tecnológicas, segundo apontado por Amorim [4] é muito difícil obter na prática industrial as condições ótimas de usinagem e os mesmos resultados informados pelas tabelas tecnológicas fornecidas pelos fabricantes. Isso ocorre tanto pela complexidade na fabricação de moldes e matrizes quanto pela diferença entre os materiais efetivamente empregados na fabricação e aqueles que foram usados pelos fabricantes para a elaboração das tabelas tecnológicas de controle dos equipamentos de usinagem. O cálculo da medida final do eletrodo para o desbaste $\left(\mathrm{M}_{\mathrm{fd}}\right)$ e para o acabamento $\left(\mathrm{M}_{\mathrm{fa}}\right)$ é dado por [11-12]:

$$
\begin{array}{r}
M_{f d}=M_{n}-(2 G A P+2 r+C S) \\
M_{f a}=M_{n}-(2 G A P+2 r)
\end{array}
$$

Nestas fórmulas: "GAP" é a distância entre o eletrodo e a peça (no caso $284 \mu \mathrm{m}$ ), "r" a rugosidade (no caso $34 \mu \mathrm{m}$ ) e "CS" o coeficiente de segurança.

Tanto as fórmulas utilizadas para dimensionamento do eletrodo ferramenta, quanto as tabelas tecnológicas fornecidas pelos fabricantes não levam em conta o tratamento térmico das peças. Entretanto, observou-se durante os testes realizados que a medida final dos corpos de prova temperados e revenidos apresentaram uma variação próxima a $0,02 \mathrm{~mm}$ em relação às peças sem tratamento. Esta variação dimensional pode ser justificada em função dos efeitos que as transformações de 
fases no estado sólido, ocorridas no tratamento térmico, têm sobre a microestrutura dos materiais (aços), afetando suas propriedades físicas [13-14].

\section{CONCLUSÃO}

Nos ensaios realizados pôde-se observar que o tratamento térmico influencia nas dimensões finais de peças usinadas pelo processo de eletroerosão. Esta variação pode afetar as dimensões finais de moldes e matrizes e consequentemente na qualidade final do produto. Também foi constatado que as tabelas técnicas e as fórmulas conhecidas, usadas para a regulagem dos parâmetros de usinagem, não distinguem o tratamento térmico e a dureza das peças a serem usinadas.

Diante da evidência de que o eletrodo ferramenta usado em peças temperadas e revenidas não pode ter a mesma dimensão que os usados em peças não temperadas, fica a possibilidade de um estudo futuro para a determinação de um coeficiente a ser incluído nas fórmulas de dimensionamento de eletrodos que corrija esta variação na medida final da peça.

\section{Agradecimentos}

Os autores expressam seus agradecimentos ao apoio recebido pelos funcionários do SENAI Manuel Garcia Filho durante a fase de preparação e usinagem dos Corpos de Prova empregados neste trabalho.

\section{REFERÊNCIAS}

1 LIMA R.Md. Utilização de Fluidos Dielétricos à Base de Óleos Vegetais na Usinagem. Dissertação de Mestrado. 2008: p. 104.

2 JOSHI A, KOTHIYA P. Investigating effects of process variables on MRR in EDM. International Journal on Theoretical and Applied Research in Mechanical Engineering (IJTARME). 2012: p. 6.

3 FERES F. Eletroerosão Possibilita Acabamentos Altamente Precisos. O mundo da Usinagem. 2010 março; 1(3).

4 AMORIM FL. Tecnologia de Eletroerosão por Penetração na Liga de Alumínio AMP 8000 e da Liga de CuBe para Ferramentas de Moldagem de Materiais Plásticos. 2002. Tese de Doutorado. 202: p. 156.

5 SANTOS RFd. Influência do Material da Peça e do Tratamento Térmico na Eletroerosão dos Aços AISI H13 e AISI D6. 2007. Tese de Mestrado. 2007: p. 122. OLINIKI R. Influência da Combinação entre os Parâmetros de Usinagem por Eletroerosão na Integridade Superficial do Aço H13 Temperado e Revenido. 2009. Tese de Mestrado. 2009: p. 127.

7 ELETRO. Manual de Operação, Instalação e Manutenção. 2010. Apostila

8 HEXAGON METROLOGY. Micro Hite 3D - Máquina de Medição por Coordenada. Catálogo. 2015. Disponível em http://www.hexagonmetrology.com.br/TESA-Micro-Hite3D_134.htm <Acesso em 21/09/2015>.

9 ASS̄OCIAÇÃO BRASILEIRA DE NORMAS TÉCNICAS. NBR NM 122-1: Aços ferramentas. Parte 1: Classificação, designação e composição química. Rio de Janeiro, 2004.

10 ASSOCIAÇÃO BRASILEIRA DE NORMAS TÉCNICAS. NBR NM 87: Aço carbono e ligados para construção mecânica - Designação e composição química. Rio de Janeiro, 2004.

11 SENAI S. Operador de Eletroerosão por Penetração. 2010. Apostila.

12 SANTOS I. Análise dos Parâmetros de Entrada na Eletroerosão por Penetração na Liga de Titânio TI-6AL-4V. Tese de Mestrado. 2010: p. 126. 
13 ASKELAND DR, Phulé PP. Ciência e Engenharia dos Materiais. 1st ed. São Paulo: Cengage Learing; 2008.

14 REBECHI JG. Correlações Numéricas entre Taxas de Resfriamento, Microestrutura e Propriedades Mecânicas para o Tratamento Térmico dos Aços AISI/SAE 4140. Tese de Mestrado. 2011: p. 92. 